\title{
Introduction to Minitrack on AI, Machine Learning, IOT \& Analytics: Exploring The Implications For Knowledge Management And Innovation
}

\author{
Ron Freeze \\ University of Arkansas \\ rfreeze@walton.uark.edu
}

\author{
Rhonda Syler \\ University of Arkansas \\ rsyler@walton.uark.edu
}

\begin{abstract}
Welcome to the HICSS-52 Minitrack on AI, Machine Learning, IOT \& Analytics where we set out to explore these emerging technologies and trends from the lense of knowledge management and innovation. It is from this perspective we seek to understand the implications of the deployment and application of these technologies on knowledge management and creation. This minitrack includes three papers. Two papers explore conceptual issues in the artificial intelligence space with one looking specifically at understanding machine language in the context of artificial intelligence laying a foundation for discussion and an operationalization of terms. The second paper then uses AI and visualization as a methodology to explore research questions, specifically the impact of managerial response on customer reviews. The third and final paper of the minitrack shifts gears to explore the effectiveness of an IOT solution in 10 Finnish small-to-medium sized enterprises (SME). Together the three papers combine to provide insight into the implications of machine learning, artificial intelligence, and IoT in the knowledge management and innovation domain.
\end{abstract}

\section{Introduction}

The exponential growth of data-intensive technologies such as IoT, IoMT, augmented reality, machine learning applications, and artificial intelligence is creating a rich landscape for the collection, organization, storage, and dissemination of knowledge. The implications of the impact these technologies have on the knowledge management ecosystem include process integration issues, data storage and data management challenges, behavioral issues such as trust in output from these technologies, and even challenges in the analytics process. Additionally, understanding the potential impact of these systems helps inform how to build and use the infrastructures and processes to achieve improved decision making and organizational performance. This minitrack seeks a focus on studies that contribute to the understanding of the characteristics of these artifacts and the challenges they present in the context of knowledge management and knowledge creation.

\section{Machine Learning in Artificial Intelligence: Towards a Common Understanding (Kühl, Goutier, Hirt \& Satzger)}

In this paper, Kühl, Goutier, Hirt, and Satzger explore the role machine learning plays within artificial intelligence. The authors discuss the growing prevalence of machine learning and artificial intelligence. The authors first differentiate between machine learning and artificial intelligence before dissecting the contribution of machine learning to artificial intelligence.

More specifically, Kühl et al. provide a review of relevant literature and present a conceptual framework that clarifies the role of machine learning to build (artificial) intelligent agents. The primary contributions and take-aways from this paper include the operationalization of the constructs of machine learning and artificial intelligence.

\section{Artificial Intelligence and Visualization: A Deep-Learning Approach to Analyze Hotel Reviews \& Responses (Ku, Chang, Wang, Chen \& Hsiao)}

This paper explores artificial intelligence and visualization to study the managerial response to online hotel reviews and the impact on purchasing decisions. According to the authors $\mathrm{Ku}$, Chang, Wang, Chen, and Hsiao, as the practice and presence of online reviews becomes more prevalent, consumers are relying on them more and more to make purchasing decisions. This reliance of the customer establishs the importance of exploring the topic. More specifically, the authors seek to explore the impact of managerial responses on consumer use of online hotel reviews. To study this 
question, $\mathrm{Ku}$ et al. deploy a framework that integrates visual analytics and machine learning techniques. In addition to examining if hotel managers respond to positive and negative reviews differently, the authors then use a deep-learning approach to prioritize responses.

\section{Findings from Multipurpose IoT Solution Experimentations in Finnish SMEs: Common Expectations and Challenges (Vermanen \& Harkke)}

Finally, in the third paper, Vermanen \& Harkke explore the effectiveness of IoT innovations in 10 Finnish small to medium enterprises (SMEs). The SME segment, according to the authors, are "showing increasing interest in modern digital solutions in hopes of streamlining their work processes." Despite these efforts, these SMEs are simultaneously struggling to adequately resource the endeavors. In addition, they are challenged with understanding the application of the IoT solutions to their business environment. To help facilitate the technical transition, Vermanen and Harkke's study provides 10 Finnish SMEs with multipurpose IoT solutions and then allow them to conduct guided solution processes. The line of business of the participating companies varied widely from manufacturing to transportation and hospitality. In addition, the individual needs and expectations towards IoT varied among the participants. The companies' initial expectations were compared to actual results with the objective of identifying the salient challenges of the IoT deployment solutions for SMEs in general. While the findings are limited in generalizability, the authors provide insight on how the specific IoT solutions can better serve SMEs.

\section{Conclusion}

This minitrack could not be successful without the research efforts of the authors. We are grateful for the authors' contributions to advancing our understanding of the phenomena surrounding emerging trends of AI, IoT, machine learning, and analytics on knowledge management and innovation. It is through these insights that we are able to contribute to the field. We anticipate these issues continuing to be a salient area of inquiry. We wish the authors the best with your ongoing research and invite you to join us at HICSS again next year. 